\title{
Engineering antiferromagnetic Heisenberg spin chains for maximizing of the groundstate entanglement
}

\author{
R. Xin ${ }^{1}$, Z. Song ${ }^{1, a}$ and C. P. Sun ${ }^{1,2, a, b}$ \\ ${ }^{1}$ Department of Physics, Nankai University, Tianjin 300071, China and \\ 2 Institute of Theoretical Physics, The Chinese Academy of Science, Beijing, 100080, China
}

\begin{abstract}
We study the correlation function and concurrence for the eigenstates with zero spin of engineered Heisenberg models to explore the entanglement property. It is shown that the total nearest neighbor (NN) correlation function of zero-spin eigenstates reaches its local extremum when the coupling strength is uniform, and correspondingly the groundstate entanglement of $d$-D cubic AF Heisenberg model is locally maximized. Moreover, numerical calculations for a $N$-site quantum spin ring with cosinusoidally modulated exchange coupling, i.e. $J_{i}=J(1+\cos (2 \pi i / N))$, indicate that the uniform coupling is not the unique optimal distribution for maximizing the groundstate entanglement and this modulation of interactions can induce the longer range entanglement.

PACS numbers: $\quad$ 03.65.Ud, 75.10.Jm
\end{abstract}

Introduction. Studies of various quantum spin models are very important in understanding the properties of realistic solid state systems. Numerical and analytical studies have shown that the groundstate properties are sensitive to the geometry of the lattice $1,2,3,4,5$ and the distribution of the exchange couplings between two neighbor spins [6, 7, [8, 9]. Although much effort has already been made to investigate the properties of the ground state, the exact result about it is still rare. For instance, a spin-1/2 Heisenberg ring is one of the nontrivial, relatively simple physical systems. It was the first one dimensional quantum model solved exactly by a straightforward diagonalization of the Hamiltonian and has been used as a testing ground for many theoretical approaches. Bethe found the eigenvalues and eigenfunctions of the corresponding Hamiltonian [10], but the obtained exact eigenfunctions are so complicated that they are almost useless for calculating some physical quantities, such as something relevant to correlation functions, to reveal all the characteristic properties of the ground state. Recently, quantum entanglement was proposed as a new type of physical resource, which is desired to depict the features of the ground state, like the correlation function or other conservative quantities. It was found that the ground state of an AF Heisenberg ring possesses maximal entanglement compared to that of excited states 11] and the entanglement is believed to have somthing to do with the quantum phase transitions [12].

The present paper explores the pairwise entanglement of the zero-spin ground state of the Heisenberg model with certain engineered inhomogeneous coupling constants. We first make a simple observation that the total nearest neighbor (NN) correlation function of zero-spin eigenstate (with vanishing components of total spin) is locally maximized for the uniformly distributed coupling strengths, while the groundstate entanglement of the $d$ $\mathrm{D}$ cubic antiferromagnetic (AF) Heisenberg model can locally maximize in this case, correspondingly. However, the uniform distribution of coupling constants is not the unique optimal one for maximizing the groundstate entanglement in the case with engineered coupling constants. Namely, the homogeneity of couplings is only the sufficient conditions rather than a necessary condition in maximizing the groundstate entanglement. To demonstrate this point of view, we study the $N$-site ring system with cosinusoidally modulated exchange coupling, i.e., $J_{i}=J(1+\cos (2 \pi i / N))$ in both analytical and numerical approaches. We discovered that the ground state with varying couplings $J_{i}$ and that with the fixed coupling $J$ are nearly identical.

Maximization of the groundstate entanglement. The Hamiltonian $H=\sum_{\langle i j\rangle} J_{i j} \mathbf{S}_{i} \cdot \mathbf{S}_{j}$ of Heisenberg spin model can be written in terms of the spin operator $\mathbf{S}_{i}$ at $i$ th site, where $J_{i j}$ is the coupling constant of exchange interaction, which is trivially restricted to be nonzero in this paper. Our studies will focus on those states with vanishing components of total spin, or called the zero spin states. In this sense the relationship between correlation and concurrence has been well established [13]. To describe the behavior of correlation one can define the nearest neighbor $(\mathrm{NN})$ correlation function

$$
F_{0}\left(J_{i j}\right)=\frac{1}{N} \sum_{\langle i j\rangle}\left\langle\mathbf{S}_{i} \cdot \mathbf{S}_{j}\right\rangle_{0}
$$

for the eigenstate $\left|\psi_{0}\right\rangle$ with zero spin. The sandwich $\left\langle\mathbf{S}_{i} \cdot \mathbf{S}_{j}\right\rangle_{0}=\left\langle\psi_{0}\left|\mathbf{S}_{i} \cdot \mathbf{S}_{j}\right| \psi_{0}\right\rangle$ is defined as the expectation value of $\mathbf{S}_{i} \cdot \mathbf{S}_{j}$ in the zero-spin eigenstate $\left|\psi_{0}\right\rangle$. Formally, it is proportional to the average of the Hamiltonian by assuming the identical coupling constants. What we concern is the $J_{i j}$-dependent behavior of the correlation function, which can be characterized by the extremum of $F_{0}\left(J_{i j}\right)$.

Now, for a zero-spin eigenstate, we show that $F_{0}\left(J_{i j}\right)$ can reach its extremum when all the exchange constants are identical, $J_{i j}=J$, if $F_{0}\left(J_{i j}\right)$ is analytical at this point. 
Actually, the eigen energy can be written as

$$
E_{0}=\langle H\rangle_{0}=\sum_{\langle i j\rangle} J_{i j}\left\langle\mathbf{S}_{i} \cdot \mathbf{S}_{j}\right\rangle_{0} .
$$

Differentiating the above equation with respect to an arbitrary coupling strength $J_{k l}$, one can get $\partial E_{0} / \partial J_{k l}=$ $\sum_{\langle i j\rangle} J_{i j} \partial\left\langle\mathbf{S}_{i} \cdot \mathbf{S}_{j}\right\rangle_{0} / \partial J_{k l}+\left\langle\mathbf{S}_{k} \cdot \mathbf{S}_{l}\right\rangle_{0}$. On the other hand, together with the Feynman-Hellman theorem

$$
\frac{\partial E_{0}}{\partial J_{k l}}=\left\langle\frac{\partial H}{\partial J_{k l}}\right\rangle_{0}=\left\langle\mathbf{S}_{k} \cdot \mathbf{S}_{l}\right\rangle_{0}
$$

it indicates that $\sum_{\langle i j\rangle} J_{i j} \partial\left\langle\mathbf{S}_{i} \cdot \mathbf{S}_{j}\right\rangle_{0} / \partial J_{l k}=0$. Obviously, when all the coupling strengths $J_{i j}=J$, we get $\partial F_{0} / \partial J_{i j}=0$, which means that $F_{0}$ has an extremum for the uniform coupling strength. Notice that this conclusion is always true no matter the system is bipartite or non-bipartite lattice and the state is ground state or excited state. However, $J_{i j}=J$ may not be the unique distribution for $F_{0}$ to reach the extremum. We will find that there may exist a periodic coupling strength distribution in 1-D system which induces the same ground state approximately as that with uniform coupling distribution.

Now we apply the above conclusion to the bipartite lattice with $J_{i j}>0$ and $N_{A}=N_{B}$, where $N_{A}, N_{B}$ are the numbers of the sites belonging to sublattices $A$ and $B$. According to Lieb's theorem [14], the ground state is singlet and has $S=0$, which ensures that $F_{g}$ reaches its minimum $F_{g}(1)=E_{g} / N$ at the point $J_{i j}=1$, where the zero-spin state $\left|\psi_{0}\right\rangle$ is replaced by the ground state $|g\rangle$. Furthermore, we consider the groundstate entanglement for the AF Heisenberg model on a $d$-D cubic lattice with translational symmetry in all directions. Here the symmetry specifies the geometry of the lattice only, i.e., the Hamiltonian may not have the translational symmetry. Since the upper bound of $E_{g}$ is $-\sum_{\langle i j\rangle} 1 / 4$ in the vicinity of the point $J_{i j}=1$, we have the inequality $F_{g}(1)<-(1 / N) \sum_{\langle i j\rangle} 1 / 4$ from $\left\langle\mathbf{S}_{i} \cdot \mathbf{S}_{j}\right\rangle_{g}<-1 / 4$. On the other hand, the pairwise concurrence for such system is $C_{g}=(1 / 2) \max \left\{-(4 / N) \sum_{\langle i j\rangle}\left(\left\langle\mathbf{S}_{i} \cdot \mathbf{S}_{j}\right\rangle_{g}-1\right), 0\right\}=$ $(1 / 2) \max \left\{-\left[4 F_{g}(1)+(1 / N) \sum_{\langle i j\rangle} 1\right], 0\right\}$ [13], or

$$
C_{g}=-\frac{1}{2}\left[4 F_{g}(1)+\frac{M}{N}\right]
$$

which maximizes in the vicinity of the point $J_{i j}=1$, where $M=\sum_{\langle i j\rangle} 1$ is the link numbers. Therefore we get the conclusion that the ground state has locally maximal pairwise entanglement when the exchange interactions distribute uniformly. A similar conclusion has been obtained for the $X X Z$ model at the isotropic point [15, 16].

Notice that the above statement does not mean that the concurrence is the maximum in the whole range of coupling constants, but just in the vicinity of the uniform point. In order to illustrate this, we investigate a simple spin model, the AF Heisenberg ring with alternative coupling constant. The Hamiltonian of $N$-site ring is

$$
H=\sum_{i \in \text { odd }} \mathbf{S}_{i} \cdot \mathbf{S}_{i+1}+\sum_{i \in \text { even }} J \mathbf{S}_{i} \cdot \mathbf{S}_{i+1} .
$$

It is well known that the value of the $N N$ pairwise concurrence is 0.386 for $J=1$ 13]. On the other hand, in the limit cases of $J=0$ and $\infty$, the exact ground states are $\phi_{1}=[12][34] \ldots \ldots[N-1 N]$, and $\phi_{2}=[23][45] \ldots \ldots[N 1]$ respectively, where $[i j]$ denotes a resonant valence bond (RVB) of two spins located at the lattice sites $i$ and $j$ [17]. Both $\phi_{1}$ and $\phi_{2}$ has a same concurrence 0.5 , which is larger than that of the state at uniform point. This demonstrates that the concurrence is not the maximum in the whole range of coupling constants. The groundstate concurrences for small systems with $N=8,10$ and 12 are calculated by exact diagonalization method and plotted in Fig.1. It indicates that the concurrences takes its local maximum at the uniform point.

Cosinusoidally modulated systems. As mentioned in advance, the uniform system may not be the unique optimal one possessing a maximal groundstate entanglement. A simplest case is that there may exist a model with non-uniform coupling strength distribution, which has the same ground state as that of the uniform one. We now show this observation in the $X Y$ spin ring exactly and the $X X X$ spin ring approximately.

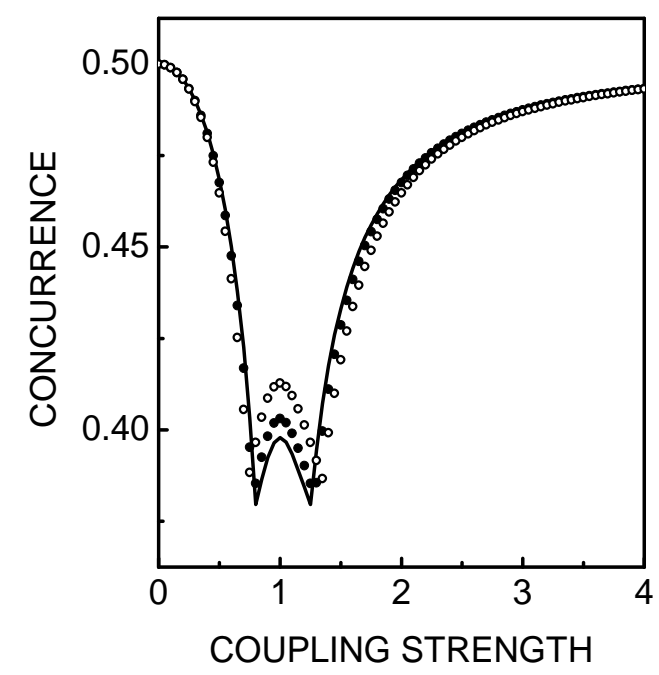

FIG. 1: The groundstate concurrences vs the coupling strength for the alternative Heisenberg ring systems with $N=8$ (circle), 10 (solid circle) and 12 (solid line). It shows that the uniform coupling point is the local maximum.

We consider the two cases of spin- $1 / 2$ Heisenberg model with the anisotropy parameter $\Delta=0,1$ on an $N$ site ring. With the cosinusoidally modulated exchange couplings, i.e. $J_{i}=J(1+\cos (2 \pi i / N))$, the Hamiltonian 
$H=H_{0}+H_{a d d}$ can be separated into two parts

$$
H_{0}=J \sum_{i}^{N}\left(S_{i}^{x} S_{i+1}^{x}+S_{i}^{y} S_{i+1}^{y}+\Delta S_{i}^{z} S_{i+1}^{z}\right),
$$

and

$$
H_{a d d}=J \sum_{i}^{N} \cos \left(\frac{2 \pi i}{N}\right)\left(S_{i}^{x} S_{i+1}^{x}+S_{i}^{y} S_{i+1}^{y}+\Delta S_{i}^{z} S_{i+1}^{z}\right) .
$$

where $2 \pi i / N$ is a factor to determine the profile of the additional Hamiltonian. $H$ was a pure toy model before the array of quantum dots is considered as a media to

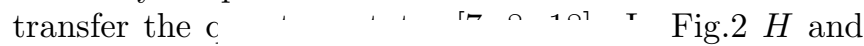
$H_{0}$ are illustr has a subtle $r$ see that $H_{a d d}$ nd states.

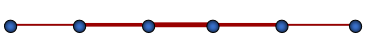

(a)

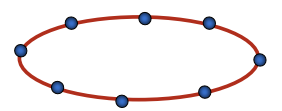

(b)

FIG. 2: Schematic illustration of cosinusoidally modulated (a) and uniform (b) NN coupling spin ring systems. The two most separated sites in (a) is the nearest neighbor in (b).

The simplest case $\Delta=0$ is first considered as the so called $X Y$ model or the hardcore boson model. It is well known that if $N / 2$ is odd the spectrum of the $X Y$ Heisenberg spin ring in the subspace with $S_{z}=0$ is reduced to the model of non-interacting spinless fermion system. The reduced Hamiltonian consists of two parts $H_{0}^{X Y}=(J / 2) \sum_{i}^{N}\left(a_{i}^{\dagger} a_{i+1}+h . c\right)$ and $H_{a d d}^{X Y}=(J / 2)$ $\sum_{i}^{N} \cos (2 \pi i / N)\left(a_{i}^{\dagger} a_{i+1}+h . c\right)$, written respectively in terms of the spinless fermion operata $a_{i}^{\dagger}$ at $i$ th site. In $k$ space, by using the discrete Fourier transformation $a_{l}^{\dagger}=$ $1 / \sqrt{N} \sum_{l}^{N} a_{k}^{\dagger} \exp (i k l),(k=2 \pi n / N, n=0, \pm 1, \pm 2, \ldots$, $\pm(N / 2-1), N / 2)$, they can be re-written as

$$
H_{0}^{X Y}=J \sum_{k} \cos k a_{k}^{\dagger} a_{k}
$$

and

$$
H_{a d d}^{X Y}=\frac{J}{2} \sum_{k}\left[e^{-i \frac{\pi}{N}} \cos \left(k+\frac{\pi}{N}\right) a_{k}^{\dagger} a_{k+\frac{2 \pi}{N}}+h . c\right] .
$$

The physics of the above Hamiltonian is quite obvious. The additional Hamiltonian $H_{\text {add }}^{X Y}$ describes an $N$-site chain system with nearest neighbor hopping in $k$ space. Notice that the $k$-dependent hopping integral $\propto \cos \left(k+\frac{\pi}{N}\right)$ vanishes at points $k= \pm k_{f}$, where $k_{f}=\pi / 2-\pi / N$ is the "fermi point" ( surface ) for the one dimensional half-filled spinless fermion model $H_{0}^{X Y}$. It is easy to find that the particles are confined either in the regions $|k| \leq k_{f}$ or $|k|>k_{f}$. Actually, it is easy to prove that the particle number in the region $|k| \leq k_{f}$ ( or $\left.|k|>k_{f}\right)$ is a good quantum number for $H_{a d d}^{X Y}$, i.e., $\left[\mathbf{n}, H_{\text {add }}^{X Y}\right]=0$, where $\mathbf{n}=\sum_{k \in\left\{|k| \leq k_{f}\right\}} a_{k}^{\dagger} a_{k}$.

Obviously, there are only four eigenstates of $H_{0}^{X Y}$ with zero eigenvalues: the groundstate $\left|\psi_{g}^{X Y}\right\rangle=\prod_{|k| \leq k_{f}} a_{k}^{\dagger}|0\rangle$ and the eigenstate $\left|\psi_{\max }^{X Y}\right\rangle=\prod_{|k|>k_{f}} a_{k}^{\dagger}|0\rangle$ of $H_{0}^{X Y}$ with maximum eigenvalue. These two states indicate that the two regions in $k$-space separated by fermi points are fully filled respectively. If all the $k$-space is fully filled or empty, one can get other two eigenstates $\left|\psi_{F M}\right\rangle$, being the saturated ferromagnetic states with all spin up or down. These four eigenstates are also the eigenstates of any linear combinations of $H_{0}^{X Y}$ and $H_{a d d}^{X Y}$. Here we only consider the simplest case $H^{X Y}=H_{0}^{X Y}+H_{\text {add }}^{X Y}$, that is

$$
H^{X Y}=2 J \sum_{i}^{N} \cos ^{2}\left(\frac{\pi i}{N}\right)\left(a_{i}^{\dagger} a_{i+1}+h . c\right) .
$$

When $N / 2$ is even, the $X Y$ model is also equivalent to the hardcore boson system. But we can not get the same analytical result as that in the case of odd number of $N / 2$. Although numerical results for small $N$-site system show that the same conclusion is also true, we can not give an exact proof at present stage. Anyway, this sample implies that the factor $2 \pi i / N$ is a characteristic groundstate property for the spin ring system.

Finally we turn to the isotropic case, i.e. $\Delta=1$, in which the Hamiltonians read as $H_{0}=J \sum_{i}^{N} \mathbf{S}_{i} \cdot \mathbf{S}_{i+1}$ and $H_{a d d}=J \sum_{i}^{N} \cos (2 \pi i / N) \mathbf{S}_{i} \cdot \mathbf{S}_{i+1}$. Correspondingly we have

$$
H=H_{0}+H_{a d d}=2 J \sum_{i}^{N} \cos ^{2}\left(\frac{\pi i}{N}\right) \mathbf{S}_{i} \cdot \mathbf{S}_{i+1} .
$$

For $J<0$, it is easy to find that the ground states of $H_{0}$ and $H$ are both saturated ferromagnetic with the same eigen energy $N J / 4$. The saturated ferromagnetic state is also the eigenstate of $H_{a d d}$ with zero eigenvalue.

For $J>0$, the saturated ferromagnetic state is also the common eigenstate of $H_{0}, H_{a d d}$ and $H$. So part of the exact conclusion obtained with $X Y$ model can be extended to the isotropic antiferromagnetic Heisenberg spin ring trivially. For the ground state, we can not get similar analytical result as that in $X Y$ model. In order to investigate the relation between the ground states of $H$ and $H_{0}$, exact diagonalization is performed to compute the groundstate energies and the overlap of the two groundstate wavefunctions. In Table 1, the numerical results are listed for the systems of site number $N=12,14,16,18,20,22$ and 24 . It shows that the over- 
lap approaches the unity, i.e. the ground states of these two Hamiltonians are approximately identical.

\begin{tabular}{ccc}
\hline \hline$N$ & $\Delta E$ & Overlap \\
\hline 12 & $2.5 \times 10^{-5}$ & 0.999988 \\
14 & $2.6 \times 10^{-5}$ & 0.999983 \\
16 & $2.5 \times 10^{-5}$ & 0.999978 \\
18 & $2.4 \times 10^{-5}$ & 0.999974 \\
20 & $2.3 \times 10^{-5}$ & 0.999971 \\
22 & $2.2 \times 10^{-5}$ & 0.999967 \\
24 & $2.0 \times 10^{-5}$ & 0.999964 \\
\hline
\end{tabular}

Table 1

Table 1. The differences of groundstate energies and the overlaps of corresponding eigenfunctions of the Hamiltonian $H$ and $H_{0}$ for the $N$-site systems obtained by exact diagonalization. It shows that the ground states of the two Hamiltonians are approximately identical.

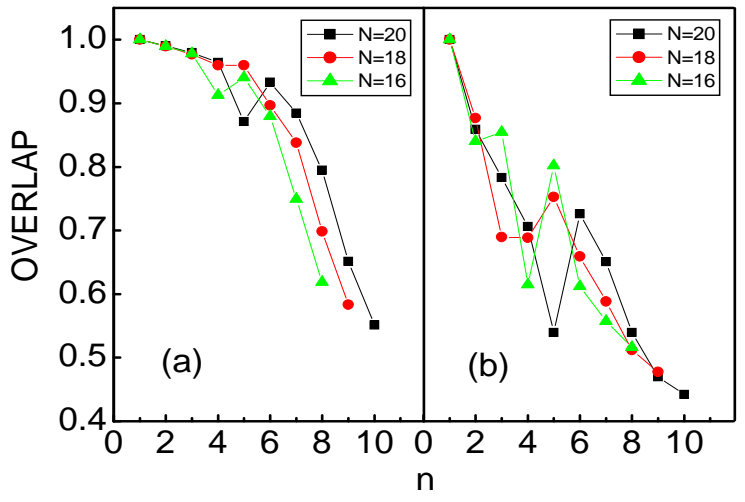

FIG. 3: The overlaps between the groundstate wavefunctions of $H_{0}$ and $H_{a d d}$ with the coupling strength $J_{i}=J(1+$ $0.5 \cos (2 n \pi i / N))(a)$ and $J_{i}=J(1+0.95 \cos (2 n \pi i / N))$ (b) for $N=20,18$ and 16 . It shows that only $2 \pi i / N$ matches the ground state of uniform coupling system.

The above results imply that the homogeneity of couplings is not the unique optimal distribution for the translational invariant groundstate having maximal entanglement. Now we investigate the ground states of the $N$-site ring systems with the coupling strength $J_{i}=$ $\left.J+J^{\prime} \cos (2 n \pi i / N)\right)$, where $n=0,1,2, \ldots, N-1$. Here $J^{\prime}<J$ ensures $J_{i}>0$ in order to avoid the degeneracy of the ground states 14]. Obviously, for $X Y$ model the conclusion for ground state obtained above is no longer available for $n \neq 0,1$. For isotropic Heisenberg model, a small size ring is investigated by numerical method. In Fig.3, the overlap of the corresponding eigenfunctions are plotted. It shows that $2 \pi / N$ is the characteristic factor for the isotropic Heisenberg ring system. On the other hand, from Fig.2 we can see that the concurrence of spins at the two ends of a modulated chain should be close to that of NN spins on a uniform ring.

Summary. In summary, the correlation and entanglement of the zero-spin eigenstates of the Heisenberg models are studied. We find that the total NN correlation function of zero-spin eigenstate reaches its local extremum when all coupling strengths are identical. Applying this fact to $d$-D cubic AF Heisenberg model, the groundstate concurrence, the measure of entanglement is locally maximized at the same point. Numerical calculations are employed to investigate a $N$-site quantum spin ring with cosinusoidally modulated exchange couplings. It indicates that the homogeneity of couplings is not the unique optimal distribution for maximizing the groundstate entanglement and this modulation of interactions can indeed result a longer rang entanglement. Furthermore, it also implies that $2 \pi / N$ is the characteristic factor for the isotropic Heisenberg ringe system.

This work is supported by the NSFC and the Knowledge Innovation Program (KIP) of the Chinese Academy of Sciences. It is also founded by the National Fundamental Research Program of China with No. 2001 CB309310.

[a] emails: songtc@nankai.edu.cn and suncp@itp.ac.cn

[b] Internet www site: http://www.itp.ac.cn/ suncp

[1] E. Lieb and D. Mattis, Phys. Rev. 125, 164 (1962).

[2] E. Dagotto, J. Riera, and D. Scalapino, Phys. Rev. B45, 5744 (1992).

[3] S. P. Strong, and A. J. Millis, Phys. Rev. Lett. 69, 2419 (1992).

[4] T. Barnes et al., Phys. Rev. B47, 3196 (1993).

[5] Magnetic systems with competing interactions: frustrated spin systems, edited by H. T. Diep (World Scientific, Singapore, 1994).

[6] K. Hida, Phys. Rev. B45, 2207 (1992).

[7] M. Christandl, N. Datta, and J. Landahl, Phys. Rev. Lett. 92, 187902 (2004).

[8] T. Shi, Ying Li, Z. Song, C. P. Sun, quant-ph/0408152

[9] Y. Li, T. Shi, B. Chen, Z. Song, C. P. Sun, quant-ph/0406159

[10] H. A.Bethe, Z. Phys. 71, 205 (1931).

[11] X. G. Wang, quant-ph/0406182

[12] S-J Gu, S-S Deng, Y-Q Li and H-Q Lin, Phys. Rev. Lett 93, 086402 (2004).

[13] Kevin M. O'Connor and William K. Wootters, Phys. Rev. A 63052302 (2001).

[14] E. H. Lieb and D. C. Mattis, J. Math. Phys. 3, 749 (1962); E. H. Lieb, Phys. Rev. Lett. 62, 1201 (1989).

[15] M-F Yang, quant-ph/0407226

[16] S-J Gu, G-S Tian and H-Q Lin, quant-ph/0408101

[17] P.W. Anderson, Mater. Res. Bull. 8, 153 (1973).

[18] B. E. Kane, Nature 393, 133 (2003); D. Loss, D. P. DiVincenzo, Phys. Rev. A 57, 120 (1998). 